\title{
Shared Decision Making im allgemeinpsychiatrischen Akutsetting
}

\author{
Eine cluster-randomisierte Studie in der Behandlung \\ der Schizophrenie (SDMPLUS)
}

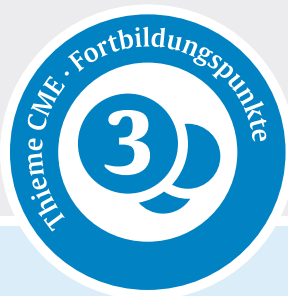

Stephan Heres ${ }^{1}$, Fabian Holzhüter ${ }^{2}$, Johannes Hamann ${ }^{2}$

1 kbo-Klinik für Psychiatrie und Psychotherapie der

kbo-Tagesklinik und Institutsambulanz Nord Schwabing, München

2 Technische Universität München

\section{ZUSAMMENFASSUNG}

Ziel: Patienten, die an einer schwerwiegenden psychiatrischen Erkrankung leiden, werden von den behandelnden Psychiatern oft nicht im Rahmen einer partizipativen Entscheidungsfindung (shared decision making, SDM) mit in die Entscheidungsprozesse einbezogen. Da SDM auch die Behandlungsergebnisse in der Therapie psychiatrischer Erkrankungen verbessern könnte, untersucht die dargestellte Studie (SDMPLUS) die Anwendung von SDM im Bereich akut erkrankter Patienten.

Methodik: In dieser multizentrischen, cluster-randomisierten Studie wurde der Einsatz von SDMPLUS gegenüber der Standardbehandlung auf 12 akutpsychiatrischen Stationen in 5 Kliniken untersucht. Alle Patienten, die an einer schizoaffektiven Störung oder einer Schizophrenie litten und die Einschlusskriterien der Studie erfüllten, wurden bei Aufnahme auf die Stationen konsekutiv in die Studie eingeschlossen. Auf den Interventionsstationen erhielten sie ein Gruppentraining in den Kommunikationstechniken des SDMPLUS. Die Behandlungsteams dieser Stationen durchliefen 2 halbtägige Workshops zum Erwerb der Techniken. Auf den Kontrollstationen wurden weder Patienten noch Behandlungsteams trainiert, die Behandlung verlief unverändert („treatment as usual“, TAU). Der primäre Zielparameter der Studie war das Ausmaß der subjektiv empfundenen Einbeziehung in die Entscheidungsfindungsprozesse der Patienten nach 3 Wochen. Retrospektiv wurden in einer Post-hoc-Analyse Daten zu Aggressionshand- lungen und freiheitsentziehenden Maßnahmen nacherhoben und ausgewertet.

Ergebnis: Insgesamt wurden je 161 Patienten auf den Interventions- und Kontrollstationen in die Studie aufgenommen. Die Intervention SDMPLUS führte zu einem höheren Maß an empfundener Einbeziehung in die Entscheidungsprozesse, dargestellt durch einen mittleren Unterschied von 16,5 Punkten in der SMD-Q-9-Skala. Darüber hinaus waren die therapeutische Allianz, die Zufriedenheit mit der Behandlung und die selbstberichtete Adhärenz der Interventionspatienten höher als in der Kontrollgruppe. Allerdings fanden sich in der Nachbeobachtungsphase über die Dauer eines Jahres hinweg keine Hinweise auf eine Erhöhung der Adhärenz oder eine Reduktion der Rehospitalisierungsraten. Auch zeigte die Intervention keine direkte Auswirkung auf das Auftreten von Aggressionshandlungen oder die Anwendung von freiheitsentziehenden Maßnahmen oder Zwangsbehandlungen. Patienten mit einer dokumentierten Aggressionshandlungen oder der Aufnahme in die stationäre Behandlung entgegen ihrem Willen konnten jedoch in gleichem Ausmaß von der Intervention hinsichtlich der erlebten Einbeziehung in Entscheidungsprozesse profitieren wie die restlichen Teilnehmer der Studie.

Zusammenfassung: Die Studie konnte zeigen, dass die Kommunikationstechniken des SDMPLUS (z. B. auch das Einbeziehen von Elementen der motivierenden Gesprächsführung) eine Möglichkeit darstellen, die partizipative Entscheidungsfindung auch im akutpsychiatrischen Behandlungskontext im Interesse der Patienten einzusetzen. Die Schnittstellenproblematik zwischen der stationären und der post-stationären Behandlung muss hierbei aber künftig noch stärker in den Fokus genommen werden, um die Effekte nachhaltig zu festigen.

\section{Einleitung}

Die partizipative Entscheidungsfindung, im Englischen etwas griffiger als „shared decision making“(SDM) bezeichnet, wird von Patienten, Bezugspersonen und Psychiatern gleichermaßen als sinnvolles Vorgehen anerkannt, da erwiesener Maßen durch sie die Zufriedenheit der Patienten mit der Behandlung und die Therapieerfolge positiv beeinflusst werden können [1]. Generell kann man von einer ethischen Grundforderung sprechen, die hier erfüllt wird, denn die Einbeziehung eines Patienten in die Entscheidungsprozesse seiner Behandlung ist für jeden Therapeuten verpflichtend [2]. Dennoch wird gerade in der Psychiatrie SDM generell eher selten praktiziert [3, 4], und man findet die Anwendung im akutpsychiatrischen Setting, in dem Patienten mitunter auch gegen ihren Willen stationär aufgenommen und behandelt werden, praktisch gar nicht $[5,6]$. Neben den allgemein bekannten Umsetzungsproblemen von SDM in der Medizin, beispielsweise dem Zeitdruck im klinischen Alltag [7], scheinen darüber hinaus Faktoren eine Rolle zu spielen, die eher spezifisch für den psychiatrischen Fachbereich oder die psychiatrisch Erkrankten gelten.

Einleuchtend ist hierbei zunächst das mitunter deutlich eingeschränkte Engagement der Erkrankten, sich an einer partizipativen Entscheidungsfindung selbst aktiv zu beteiligen. Hierbei können eine krankheitsbedingte Antriebsminderung, generelle Passivität im Handeln [8], Hoffnungs- und Perspektivlosigkeit $[9,10]$ oder ein Unterlegenheitsgefühl 
in der Patient-Arzt-Beziehung [6] zugrunde liegen. Diese eingeschränkte Fähigkeit, sich aktiv am Prozess der gemeinsamen Entscheidungsfindung zu beteiligen, sehen wiederum Psychiater als eine der größten Hürden in der Anwendung von SDM in der Akutpsychiatrie, gepaart mit einer krankheitsimmanenten Schwierigkeit, die eigene Erkrankung als solche zu erkennen und die Behandlungsbedürftigkeit zu reflektieren [10-12]. Durch die Fürsorgepflicht des Behandelnden, der im besten Interesse des Erkrankten handeln sollte, und die beschriebenen Hürden entsteht ein nicht aufzulösendes Dilemma. Eine Einbeziehung des Erkrankten in die Entscheidung kann zu einem Diskussionsergebnis führen, das aus Sicht der Therapeuten nicht optimal oder gar dem Genesungsprozess gegenläufig ist, und dennoch im Sinne der partizipativen Entscheidungsfindung lege artis verlaufen ist. Folglich hält die Angst vor einem derartigen Ergebnis viele Therapeuten vom Einsatz des SDM ab, in der Annahme, so im besten Interesse des Patienten zu handeln, was aber auch als Bevormundung oder Eingriff in die Persönlichkeitsrechte eines Erkrankten verstanden werden kann oder sogar muss.

Ziel der aktuellen Studie war es, gerade im Setting einer vollstationären akutpsychiatrischen Behandlung den Prozess einer partizipativen Entscheidungsfindung proaktiv anzuleiten und bezüglich messbarer Effekte auf die Patientenzufriedenheit mit der Behandlung zu untersuchen. Hierzu wurde eine komplexe Intervention, SDMPLUS (shared decision making PLUS) [13] eingesetzt, die gezielt die beschriebenen Hürden in der Anwendung von SDM durch Schulungen von Patienten in aktiver Entscheidungsfindung sowie Behandlungsteams in motivierenden und deeskalierenden Gesprächstechniken senkt. Die komplexe Intervention soll es den Erkrankten erleichtern, eigene Präferenzen und Wünsche zu identifizieren und in die Entscheidungsfindung aktiv mit einzubringen. Auf Seiten des Behandlungsteams lag der Schwerpunkt auf der Motivation der Patienten und spezifischen Hilfestellungen, verfahrene Gesprächssituationen zu einem konstruktiveren Verlauf zu wenden. Dadurch könnte auf Seiten der Erkrankten ein höheres Maß an empfundener Einbeziehung in Entscheidungsprozesse der eigenen Therapie resultieren, was abgesehen von möglichen positiven Effekten der jeweiligen Entscheidung auf den weiteren Verlauf der Therapie per se einen Zugewinn an Behandlungszufriedenheit darstellen würde [2]. Denkbar wäre ebenso eine Reduktion von aggressivem Verhalten oder freiheitsentziehender Maßnahmen während des Aufenthaltes, was in einer Post-hoc-Analyse näher betrachtet wurde. Ein besonderes Augenmerk lag hierbei in der Gruppe derjenigen Patienten, die entgegen ihrem Willen in die Klinik aufgenommen worden waren oder bereits in der Vergangenheit aggressives Verhalten aus Sicht der Behandler gezeigt hatten. Auch über den stationären Behandlungsrahmen hinaus wären positive Effekte auf das generelle Patienten-Arzt-Verhältnis, die Beteiligung an der Gestaltung des weiteren Genesungsprozesses und die Verhinderung von Rückfällen denkbar und wünschenswert $[14,15]$.

\section{Methoden}

\section{Studiendesign, Randomisierung und Verblindung}

Die Studie wurde multizentrisch auf jeweils hinsichtlich mehrerer Parameter passend gepaarter Stationseinheiten im geschützten akutpsychiatrischen Setting in Oberbayern durchgeführt. Es beteiligten sich 5 Fachkrankenhäuser mit insgesamt 12 Stationen, die in 6 Paare mittels Cluster-Randomisierung aufgeteilt wurden [16]. Jeweils eine Station eines Paares wurde der SDMPLUS-Intervention zugeordnet, auf der anderen Station wurde die reguläre Patientenversorgung als Kontrollsetting unverändert fortgeführt (,treatment as usual“, TAU). Die bestmögliche Paarung der Stationen wurde hinsichtlich der Anzahl der behandelten Patienten, der Verteilung von Diagnosen, der Rahmenbedingung der Behandlung und des Personalschlüssels vorgenommen.

Aufgrund des gewählten Studiendesigns war es nicht erforderlich, innerhalb einer Station eine Verblindung bezüglich der angewandten Interventionen durchzuführen. Zwischen der Interventions- und Kontrollstation wurde aber striktes Stillschweigen über die vermittelten Inhalte vereinbart. Eine Verblindung bezüglich der Erhebung des primären Endpunktes der Studie war nicht möglich, da hierbei eine Selbstbeurteilungsskala an die behandelten Patienten ausgegeben wurde. Dies war auch bei den meisten sekundären Zielparametern der Fall.

\section{Studienteilnehmer}

Alle Patienten im Alter von 18 bis 65 Jahre, die unter der Diagnose einer Schizophrenie (ICD-10 F20.x) oder einer schizoaffektiven Störung (ICD 10 F25.x) aufgenommen wurden, erfüllten die Einschlusskriterien, wenn es ihnen möglich war, an einstündigen Schulungen absehbar teilzunehmen und ihr schriftliches Einverständnis zur Studienteilnahme abzugeben. Sämtlichen Patienten, die diese Kriterien erfüllten, wurde die Studienteilnahme angeboten. Lediglich diejenigen Patienten, die an einem fortgeschrittenen demenziellen Prozess litten oder sich aufgrund geringer Sprachkenntnisse nicht aktiv an einer Entscheidungsfindung in deutscher Sprache beteiligen konnten, wurden nicht berücksichtigt. Explizit wurde denjenigen Patienten die Studienteilnahme angeboten, die nicht aufgrund ihrer freien Willensentscheidung in die stationäre Behandlung aufgenommen worden waren.

\section{Intervention und Kontrollbedingung}

Die SDMPLUS-Intervention wurde von den Autoren konzipiert [13], einige Bestandteile (z. B. die Schulung der Patienten) sind bereits in vorausgegangenen Studien erprobt und beschrieben worden [18]. Die komplexe Intervention SDMPLUS unterstützt Patienten und Behandlungsteams im wechselseitigen Initiieren und gemeinsamen Führen von partizipativen Entscheidungsprozessen. Bestehende Konzepte zur Schulung von Behandlungsteams [19] wurden vor allem hinsichtlich des aktiven Einbeziehens von Patienten mit eingeschränkter oder fehlender Krank- 
heitseinsicht, aber auch Erkrankter mit eingeschränkten Fähigkeiten zur gemeinsamen Entscheidungsfindung erweitert. Techniken der motivationalen Gesprächsführung fanden ebenso Berücksichtigung wie Interventionen aus der deeskalierenden Verhandlungsführung [13].

Die beiden Studieninitiatoren JH und SH der TUM (Fachärzte für Psychiatrie und Psychotherapie) führten interaktive Workshops mit den gesamten Behandlungsteams (Pflegepersonal, Ärzte, Sozialpädagogen, Psychologen, Fachtherapeuten) der Interventionsstationen durch. Die beiden halbtägigen Workshops in den teilnehmenden Fachkrankenhäusern umfassten Vorträge zum wissenschaftlichen Hintergrund, Einführungen in die unterschiedlichen Interventionen, Fallbeispiele und zahlreiche Rollenspiele, um die Techniken einzuüben. Für das komplette ärztliche Personal war die Teilnahme an beiden Workshops verpflichtend und Voraussetzung für die Studiendurchführung, die anderen Berufsgruppen sollten in möglichst hohem Ausmaß vertreten sein. Die Ärzte wurden über den gesamten Fortgang der Studie kontinuierlich vom Studienteam in der Umsetzung der Techniken wöchentlich durch Supervision unterstützt und fortlaufend dazu angehalten, das Erworbene in die Behandlung der Patienten fortwährend einzubringen. Die Patienten wurden in einem Gruppentraining 2-mal pro Woche fortlaufend geschult. Hierbei wurden Rollenspiele und unterstützende Materialien, z. B. Fragebögen zur Vorbereitung der Visitengespräche oder Anleitungen zur Hierarchisierung der Wünsche und Präferenzen genutzt [20]. Das Programm wurde über den gesamten Ablauf der Studie hinweg in allen teilnehmenden Kliniken vom Studienpersonal der TUM (unter Umständen auch in Gegenwart von Mitgliedern des Behandlungsteams) angeboten, und es wurde sichergestellt, dass jeder Studienteilnehmer mindestens 2 der angebotenen Schulungstermine besucht hat. Die Patienten und das Personal der Kontrollstationen wurden nicht geschult und keinerlei Informationen zur Studie wurden in den betreffenden Kliniken zugänglich gemacht. Im Nachgang der Studie wurde ihnen angeboten, einen kompletten Schulungszyklus zu durchlaufen und die Durchführung der Patientengruppen kennenzulernen.

\section{Datenerhebung}

Zu 4 Zeitpunkten (Baseline; 3 Wochen nach Baseline bzw. bei Entlassung, je nachdem, was zuerst eintrat; 6 und 12 Monate nach Entlassung) wurden in gleicher Weise Daten der Interventions- und Kontrollgruppe erhoben. Darüber hinaus wurden die retrospektiv erhobenen Daten zu Aggression von Patientenseite und freiheitsentziehenden Maßnahmen 3 Zeitfenstern zugeordnet: Präinterventionsphase (stationäre Aufnahme bis Studieneinschluss), Interventionsphase (Studieneinschluss bis zur Entlassung oder Zeitpunkt 3 Wochen, je nachdem, was früher eintrat) und Postinterventionsphase (Ende der Intervention bis zur Entlassung, falls Patienten nicht zuvor entlassen worden waren).

\section{Baselinedaten}

Von allen Teilnehmern wurden anamnestische (Anzahl der stationären Voraufenthalte, Dauer der Erkrankung etc.) und soziodemografische Daten, die Diagnose und die Einschätzung der Krankheitsschwere (CGI-S) und des globalem Funktionsniveaus (GAF) bei Studieneinschluss erfasst. Ergänzend wurde die Krankheitseinsicht anhand der „Birchwood Insight Scale“ [21] und die Wahrnehmung der aktuellen stationären Einweisung mittels der „MacArthur Admission Experience Survey“ [22] beurteilt.

\section{Studienendpunkte}

\section{Primärer Studienendpunkt}

Wir definierten als primären Studienendpunkt die subjektiv empfundene Einbeziehung in die medikamentöse Therapieentscheidung während des stationären Aufenthaltes, gemessen durch die SDM-Q-9-Skala nach 3 Wochen (T1) im Studienverlauf (oder zum Zeitpunkt der Entlassung, falls diese vor Ablauf der 3 Wochen stattfand). Entsprechend der Erfahrung der Forschungsgruppe Rodenburg-Vandenbussche und Kollegen [23] mit der Anwendung der SDM-Q-9-Skala interpretierten wir einen Unterschied von 15 Punkten zwischen der Interventions- und der Kontrollgruppe als klinisch relevant.

\section{Sekundäre Studienendpunkt (3-Wochen- Messzeitpunkt)}

Den möglichen Einfluss auf die therapeutische Allianz bzw. das Patient-Therapeuten-Verhältnis beurteilten wird durch die „Helping Alliance Scale“ (Patientenversion HAS-P und Therapeutenversion HAS-C) [24]. Die Patientenzufriedenheit mit der Behandlung untersuchten wir mittels der "Questionnaire on Patients' Treatment Satisfaction" (ZUF8) und das Bestehen bzw. Fortbestehen von bisher nicht ausreichend adressierten Bedürfnissen in der Bewältigung der Aufgaben des täglichen Lebens anhand des „Camberwell Assessment of Need self-report questionnaire“ (CANSAS-P). Das Ausmaß der Adhärenz mit der aktuellen medikamentösen Behandlungsstrategie bildeten wir durch die „Medication Adherence Rating Scale“ (MARS) ab [25].

\section{Sekundäre Studienendpunkte (6- bzw. 12-Monate-Messzeitpunkt)}

Im weiteren Verlauf nach Entlassung erfassten wir jeweils das subjektive Wohlbefinden und die Lebensqualität der Patienten durch den „WHO-5 well-being index“ und die „EUROHIS-QOL“ (generic quality of life questionnaire) [26]. Ebenso wurde der weiter behandelnde Psychiater nach möglichen erneuten stationären Aufnahmen des Studienteilnehmers im Nachverfolgungszeitraum befragt.

\section{Daten zu Aggressionshandlungen und freiheitsentziehenden Maßnahmen}

In den 3 Zeitfenstern wurden die Beschreibungen der Vorkommnisse, ihre Häufigkeit und die jeweilige Dauer aus den Krankenakten extrahiert. Die Operationalisierung der Aggressionshandlungen erfolgte über die „modified overt 
- Tab. 1 Soziodemografie

\begin{tabular}{|l|l|l|l|}
\hline & Intervention (n=161) & Kontrolle (n= 161) \\
\hline Alter in Jahren (Mittelwert, Standardabweichung) & $42,1(12,9)$ & $41,4(13,6)$ \\
\hline Frauen & $84(52 \%)$ & $76(47 \%)$ & 0,61 \\
\hline Hauptdiagnose & F20: $99(61 \%)$ & F20: $115(71 \%)$ \\
\hline & F25: $52(32 \%)$ & 0,21 \\
\hline Unfreiwillige stationäre Aufnahme & Andere F2x-Diagnose: $10(6 \%)$ & Andere F2x-Diagnose: $8(5 \%)$ \\
\hline Bestehendes Betreuungsverhältnis & $61(40 \%)$ & $41(28 \%)$ \\
\hline Dauer der Erkrankung in Jahren (Mittelwert, Standardabweichung) & $94(61 \%)$ & $86(58 \%)$ & 0,03 \\
\hline $\begin{array}{l}\text { Vorausgegangene stationäre Aufenthalte (Mittelwert, Standardabwei- } \\
\text { chung) }\end{array}$ & $12,4(10,3)$ & $13,0(11,3)$ \\
\hline CGI (Mittelwert, Standardabweichung) & $6,9(6,7)$ & $7,6(7,5)$ \\
\hline GAF (Mittelwert, Standardabweichung) & $5,3(0,9)$ & $5,5(0,8)$ \\
\hline Krankheitseinsicht (Mittelwert, Standardabweichung) & $37,7(13,3)$ & $36,1(12,4)$ \\
\hline MacArthur Admission Experience (Mittelwert, Standardabweichung) & $2,5(2,0)$ & $8,0(3,1)$ \\
\hline
\end{tabular}

aggression scale" (MOAS), einer validierten Skala zur Erfassung aggressiver Handlungen und deren Schweregrad [27]. Die ursprüngliche Form der Skala unterscheidet $4 \mathrm{Ka}$ tegorien von Aggression - verbale Aggression, Aggression gegenüber Objekten, Autoaggression und Aggression gegenüber Dritten - und fasst diese in einem Summenwert zusammen. Für unsere Analyse wurde der Summenwert in dichotomer Weise betrachtet, wobei lediglich ein Wert gleich Null als Nichtauftreten von Aggression, alle anderen Werte als Auftreten von Aggression angesehen wurde.

Freiheitsentziehende Maßnahmen oder Zwangsbehandlung wurden ebenfalls dichotom erfasst, sodass jeglicher Einsatz, unabhängig von der Häufigkeit, gleichermaßen als das Kriterium erfüllend bewertet wurde. Da diesen Maßnahmen Antragsprozesse vor-/nachgeschaltet sind, waren entsprechende Aufzeichnungen in den Krankenakten verfügbar. Darüber hinaus wurde das Vorliegen einer gesetzlichen Betreuung, die Einweisung entgegen den Willen des Patienten und der Zeitpunkt des ersten unbegleiteten Ausgangs von Station nach Aufnahme erfasst.

\section{Statistische Analyse}

Der primäre Zielparameter der dargestellten Studie war der Vergleich des Mittelwertes der SDM-Q-9-Skala zum Zeitpunkt Woche 3 (bzw. bei Entlassung, je nachdem, was zuerst aufgetreten war] zwischen der Interventions- und der Kontrollgruppe. Um den Effekt der Intervention auf den kontinuierlichen primären Endpunkt abzuschätzen, verwendeten wir ein gemischtes lineares Regressionsmodell mit der Clusterzuteilung als zufälliger und der Interventionszuweisung als fixer Größe, bei dem ein Signifikanzlevel von $\alpha=5 \%$ angenommen wurde. Der Einfluss der Intervention auf die sekundären Zielparameter wurde anhand von exploratorischen Analysen beschrieben. Die kontinuierlichen sekundären Zielparameter wurden anhand eines li- nearen Regressionsmodells analysiert, entsprechend dem primären Zielparameter. Für den binären kategorischen sekundären Parameter (Rehospitalisierung) wurde wiederum ein gemischtes lineares Regressionsmodell herangezogen. Da alle Zielparameter des Beobachtungszeitraums nach der Entlassung aus der stationären Behandlung statistisch nicht signifikant waren, beschränken wir uns in diesem Bericht auf die Darstellung der wesentlichen Parameter.

\section{Registrierung der Studie und Votum der Ethikkommission}

Die Studie wurde durch die Ethikkommission der Medizinischen Fakultät der Technischen Universität München, Klinikum rechts der Isar, begutachtet. Nach dem auflagefreien Votum wurde die Studie im Oktober 2016 begonnen. Sämtliche Befragte gaben nach ausführlicher Aufklärung ihr schriftliches Einverständnis zur Teilnahme an der Studie, die beim Deutschen Register Klinischer Studien angemeldet wurde (Registrierungsnummer DRKS00010880).

\section{Ergebnisse}

Von Oktober 2016 bis März 2018 wurden insgesamt 322 Patienten in die Studie eingeschlossen.

\section{Daten bei Studieneinschluss}

Die Teilnehmer waren überwiegend weiblich, im Mittel circa 42 Jahre alt und litten zumeist an einer Schizophrenie. Die bisherige Krankheitsdauer lag bei durchschnittlich 13 Jahren und beinhaltete im Mittel 7 stationäre Aufenthalte vor der aktuellen Aufnahme. Die Krankheitsschwere wurde anhand des CGI-S mit „deutlich erkrankt“ beschrieben, das globale Funktionsniveau erreichte im Mittel einen Wert von 37 (von 100) auf der GAF-Skala ( $\bullet$ Tab. 1). Von den Studienteilnehmern wurden 103 Patienten (32\%) entgegen ihrem Willen in die Klinik eingewiesen. Bei 111 Pati- 
Mögliche Rekrutierungseinheiten: 12 Cluster = 6 Paare vergleichbarer geschützter Stationen

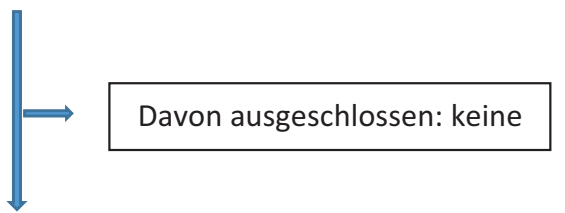

Randomisierte Rekrutierungseinheiten:

12 Cluster $=6$ Paare vergleichbarer geschützter Stationen

Der Intervention zugeordnet:

6 Cluster (Stationen), Intervention wurde von allen komplett absolviert

Mittlere Clustergröße 27 Patienten

(min/max: 22/31)

Patienten im Screening: 495

Anzahl der ausgeschlossenen Patienten:

- Ausschlusskriterium: 128

- Keine Zustimmung: 206

Patienten rekrutiert: 161

\section{"Lost to follow up"}

bis zum primären Studienendpunkt:

Kein Cluster vorzeitig verloren

32 Patienten aufgrund vorzeitiger

Entlassung ohne Messung verloren

\section{Statistisch analysierte Fälle:}

Die Daten aller Cluster wurden analysiert, primäre Endpunktanalyse bei

129 Patienten möglich
Der Kontrolle zugeordnet:

6 Clusters (Stationen), treatment as usual

(TAU) wurde aufrecht erhalten

Mittlere Clustergröße 27 Patienten

(min/max: 22/30)

Patienten im Screening: 376

Anzahl der ausgeschlossenen Patienten:

- Ausschlusskriterium: 85

- Keine Zustimmung: 130

Patienten rekrutiert: 161
"Lost to follow up"

bis zum primären Studienendpunkt:

Kein Cluster vorzeitig verloren

31 Patienten aufgrund vorzeitiger

Entlassung ohne Messung verloren

\section{Statistisch analysierte Fälle:}

Die Daten aller Cluster wurden analysiert, primäre Endpunktanalyse bei

130 Patienten möglich

- Abb. 1 Rekrutierungsübersicht (CONSORT-Diagramm)

enten fanden wir mindestens eine dokumentierte Aggressionshandlung über den gesamten stationären Aufenthalt hinweg. Freiheitsentziehende Maßnahmen ließen sich bei 76 Teilnehmern den Krankenakten entnehmen und bei 31 Patienten fand eine medikamentöse Zwangsbehandlung statt. Wie man der Darstellung des zeitlichen Verlaufs in $>$ Abb. 1 entnehmen kann, datieren die meisten Aggressionshandlungen und freiheitsentziehenden Maßnahmen auf die ersten Tage nach der stationären Aufnahme und somit in die Präinterventionsphase der Studie.

\section{Primärer Zielparameter}

Die Teilnehmer in der Interventionsgruppe fühlte sich in die Entscheidungsprozesse bezüglich der medikamentö- sen Therapie klinisch und statistisch signifikant $(p=0,002)$ mehr einbezogen als diejenigen der Kontrollgruppe. Dies konnte durch einen mittleren Unterschied von 16,5 Punkten in der SMD-Q-9-Skala gezeigt werden.

SDMPLUS zeigte keinen nachweisbaren Effekt auf das Auftreten von Aggressionshandlungen oder freiheitsentziehenden Maßnahmen während der Interventionsphase. Auch bei Patienten, die nach der Intervention weiter in der Klinik behandelt wurden, konnte in der Postinterventionsphase kein Effekt gezeigt werden. Ebenso ergab sich kein Hinweis auf einen Unterschied zwischen den Behandlungsgruppen auf die Latenz bis zum ersten Alleinausgang von Station [28]. Hinsichtlich des primären Studienendpunktes konn- 
ten unter dem Aspekt der Aggressionshandlungen fand sich ein marginal signifikanter Unterschied in der SDM-Q-9-Skala bei Patienten, die entgegen ihrem Willen in die Behandlung aufgenommen wurden versus derjenigen, die freiwillig in die Klinik gekommen waren (26, 1 vs. 12,4 ; t-test = 1,988, $d f=228, p=0,048)$. Diese Subgruppenanalyse legt nahe, dass der Effekt der SDMPLUS-Intervention auf die subjektiv erlebte Einbeziehung in Therapieentscheidungen bei unfreiwilliger Aufnahme messbar bleibt, oder in dieser Gruppe vielleicht sogar stärker ausgeprägt sein könnte.

\section{Sekundäre Zielparameter}

Auch hinsichtlich der Beurteilung des therapeutischen Verhältnisses, der Zufriedenheit mit der Behandlung und der Selbstbeurteilung der Adhärenz mit der gewählten medikamentösen Behandlungsstrategie zeigten die Patienten in der Interventionsgruppe am Beobachtungszeitpunkt T1 (3 Wochen nach Studieneinschluss bzw. zum Zeitpunkt der Entlassung, je nachdem, was zuerst eingetreten war) höhere Werte als diejenigen der Kontrollgruppe, was für alle 3 Parameter ein besseres Ergebnis darstellt[29]. Im Gegensatz hierzu konnten wir keine statistisch signifikanten Unterschiede in der Dauer des stationären Aufenthaltes, der Krankheitsschwere, dem globalen Funktionsniveau oder den Berichten zu den nicht ausreichend adressierten Bedürfnissen in der Bewältigung der Aufgaben des täglichen Lebens am Beobachtungszeitpunkt T1 finden [29].

\section{Langfristige sekundäre Zielparameter}

Während des insgesamt 12-monatigen Nachbeobachtungsintervalls zeigten sich keine Unterschiede zwischen den Gruppen bezüglich der Einschätzung der Adhärenz, der berichteten Lebensqualität und der Anzahl der stationär-psychiatrischen Aufnahmen.

\section{Diskussion}

Bisher haben mehrere Studien untersucht, wie man die partizipative Entscheidungsfindung in der Psychiatrie besser zur Anwendung bringen könnte. Es zeigten sich aber höchstens mäßige Effekte, außerdem wurden nur selten akut Erkrankte oder Patienten, die entgegen ihrem Willen in die stationäre Behandlung gekommen waren in die Studien mit einbezogen [5].

In dieser Studie kam erstmals eine komplexe Intervention zum Einsatz, in der gleichzeitig sowohl Patienten in ihren Bemühungen, an der partizipativen Entscheidungsfindung mitzuwirken als auch komplette Behandlungsteams in der deren Engagement, Erkrankten an Entscheidungsprozessen proaktiv teilhaben zu lassen, unterstützt wurden. Die Untersuchung wurde im akutpsychiatrischen Setting unter Einbeziehung auch gegen ihren Willen aufgenommener Patienten durchgeführt. Durch die komplexe Intervention konnte die von Patienten erlebte Einbeziehung in die Entscheidungsprozesse während eines stationären Aufenthaltes klinisch signifikant gesteigert werden, ebenso verbes- serte sich die therapeutische Beziehung zu den Ärzten, die Zufriedenheit mit der Therapie und (selbst eingeschätzte) Adhärenz mit der vereinbarten medikamentösen Behandlungsstrategie. Im anschließenden Nachbeobachtungszeitraum zeigten sich über ein Jahr hinweg jedoch keine Effekte auf Wiederaufnahmeraten, die berichtete Adhärenz oder die Lebensqualität der Teilnehmer. Auch konnten wir keinen Effekt auf das Auftreten von aggressivem Verhalten oder freiheitsentziehenden Maßnahmen während des stationären Aufenthaltes feststellen. Interessanter Weise prädizierte aber das Auftreten von Aggressionshandlungen und/oder freiheitsentziehender Maßnahmen nicht das Ausmaß des Effekts der Intervention, schienen diese also, zumindest in unserer Analyse, nicht zu beeinträchtigen. Auch profitierten Patienten, die unfreiwillig in die Klinik gekommen waren, mindestens im selben Ausmaß von der Intervention wie Patienten, die freiwillig aufgenommen worden waren.

\section{Stärken und Schwächen der Studie}

Während des Ablaufs der Studie wurden die Teilnehmer konsekutiv rekrutiert, um einen Selektionsbias zu verhindern. Gleichzeitig konnten sich aber weniger Patienten auf den Interventionsstationen, denen die Studienteilnahme vorgeschlagen wurde, zur Teilnahme entschließen. Dies könnte einen Einfluss auf die Ausgewogenheit des Rekrutierungsprozesses zwischen der Interventions- und der Kontrollgruppe gehabt haben. Entsprechend beobachteten wir beispielsweise eine höhere Rate von unfreiwilligen stationären Aufnahmen in der Interventionsgruppe. Dies sollte jedoch in überschaubarem Ausmaß zum Tragen gekommen sein, da die Ergebnisse der Gruppenunterschiede sowohl im primären als auch in den sekundären Zielparametern unter Berücksichtigung der Baseline-Unterschiede unverändert blieben.

Obwohl wir in wöchentlichen Kontakten mit den Interventionsstationen versuchten, die vermittelten Inhalte zu vertiefen und deren Anwendung in der Therapie der dort behandelten Patienten zu unterstützen, konnten wir das Ausmaß der tatsächlichen Umsetzung letztlich methodisch nicht standardisieren und müssen von einer gewissen Varianz in der Umsetzung der komplexen Intervention rechnen. Das ärztliche Personal konnte im Rahmen der Schulungen vollständig erfasst werden, die Einbindung der anderen Berufsgruppen erreichte in den unterschiedlichen Kliniken jedoch lediglich 10-50\% der potenziell schulbaren Mitarbeiter. Dies kann in multiprofessionell arbeitenden Behandlungsteams ebenfalls einen limitierenden Faktor in der ganzheitlichen Umsetzung der Schulungsinhalte darstellen.

\section{Diskussion der Ergebnisse}

Vorrangiges Ziel des SDMPLUS Ansatzes ist die Unterstützung der tatsächlichen Umsetzungen von partizipativen Entscheidungsprozessen in der Akutpsychiatrie im klinischen Alltag. Gerade diejenigen Patienten, die schwer erkrankt sind, eine geringe oder nicht gegebene Krankheitseinsicht mit in die Behandlung bringen oder sogar entge- 
gen ihrem eigenen Willen stationär aufgenommen worden sind, und große Schwierigkeiten in der Mitgestaltung ihrer eigenen Therapie haben, sollen von dieser komplexen Intervention profitieren können. Denn gerade diesen Patienten wird oft die Teilnahme am gemeinsamen Entscheidungsprozess per se nicht angeboten oder von den Therapeuten nicht zugetraut [11]. Die Studie sollte zeigen, dass die Anwendung von SDMPLUS-Techniken bei genau dieser Patientengruppe ein höheres Maß an erlebter Einbeziehung in Entscheidungsprozesse während eines stationären Aufenthaltes bewirken kann. Man kann nun geteilter Meinung sein, ob die bloße Einbeziehung zu einer tatsächlichen Verbesserung der therapeutischen Behandlungsergebnisse in klinischer oder gar ökonomischer Hinsicht führen kann, dennoch sehen wir 2 klar hervorzuhebende Vorteile unseres Ansatzes:

Generell haben Klienten und Patienten den nachvollziehbaren und gerechtfertigten Anspruch an das Gesundheitssystem, so auch an das psychiatrische Versorgungssystem, ihre Autonomie und ihre Würde bestmöglich während einer Konsultation oder Therapie gewahrt zu sehen [30]. SDMPLUS unterstützt diesen Wunsch. Des Weiteren unterstreichen unsere Studienergebnisse, dass Patienten trotz schwerer psychischer Erkrankung an einer partizipativen Entscheidungsfindung teilhaben können. Weder aktuelle Symptome noch Krankheitseinsicht des Patienten sollten Therapeuten davon abhalten, die Autonomie der Behandelten in Entscheidungsprozessen maximal zu wahren. Die Forschungsergebnisse von Kollegen [31], die im Einklang mit unseren aktuellen Erkenntnissen stehen, zeigen, dass dies trotz aller Vorbehalte der Therapeuten möglich ist. Abgesehen davon besteht selbstredend und allgemein anerkannt die ethische Verpflichtung, die Patientenautonomie und die Bedürfnisse der Erkrankten zu respektieren und deren Erfüllung zu unterstützen.

Unsere Studie konnte darüber hinaus zeigen, dass die Zufriedenheit mit der stationären Behandlung, das therapeutische Verhältnis und die erlebte Adhärenz mit der medikamentösen Therapiestrategie durch SDMPLUS positiv beeinflusst werden können. Dies fügt sich in den Kontext von beschriebenen Beobachtungen von Giacco und Kollegen ein, die durch intensivierte individuelle Behandlungsplanung bei unfreiwillig stationär Behandelten eine Verbesserung mehrerer weiterer Therapieziele abseits der Einbeziehung in Entscheidungsprozesse erreichen konnten [32]. In dieser Interventionsstudie waren die erzielten Effekte sogar über den Entlassungszeitpunkt hinaus weiter nachweisbar, was in unserer aktuellen Studie bedauernswerter Weise nicht gezeigt werden konnte. Möglicherweise wäre ein nachhaltigerer Effekt zu erzielen gewesen, hätte man die Therapeuten, die sich um die Fortführung der Behandlung Patienten nach der Entlassung gekümmert haben, ebenso in der SDMPLUS-Intervention geschult, und letztlich auch die Schulungsangebote für die Patienten, beispielsweise über elektronische Formate, fortgeführt.
Hinsichtlich des Auftretens von Aggressionshandlungen oder freiheitsentziehenden Maßnahmen konnte durch unsere Intervention kein Effekt nachgewiesen werden. Wir können jedoch nicht ausschließen, dass dies vor allem auch methodische Gründe hat. Die meisten derartigen Handlungen oder Maßnahmen fanden direkt nach Aufnahme, also in der Präinterventionsphase statt. Eventuell setzte also die Intervention im zeitlichen Verlauf des stationären Aufenthaltes zu spät ein, um einen Effekt auf die Vorkommnisse haben zu können. Während der Interventionsphase und in der Postinterventionsphase war die Inzidenz dieser Vorkommnisse per se sowohl in der Kontroll- als auch in der Interventionsgruppe sehr gering, was den Nachweis eines möglicherweise existierenden Effekts methodisch erschwert („floor effect“). Darüber hinaus war von Interesse, ob sozusagen in einem Umkehrschluss solche Vorkommnisse den Effekt der Intervention minimieren oder komplett aufheben. Diese Annahme wird durch Aussagen von Patienten, dass das Erleben von „Ohnmacht“ und „Ausgeliefersein“ (z. B. im Rahmen einer mechanischen Beschränkung oder Zwangsbehandlung) zu einer geringeren Beteiligung in Entscheidungsprozessen führt [23], oder die Ausübung von Zwang generell die therapeutische Allianz stört [24], untermauert. Diesen Effekt konnten wir in unsere Studie nicht erkennen. Somit kann man daraus den Schluss ziehen, dass es für eine gemeinsame Entscheidungsfindung quasi „nie zu spät“ ist.

\section{FAZIT FÜR DIE KLINISCHE PRAXIS}

Der Einsatz der komplexen Intervention SDMPLUS zeigt, dass die Teilhabe an Entscheidungsprozessen auch schwer psychisch Erkrankten, die auf geschützten Stationen und möglicherweise entgegen ihrem Willen behandelt werden, möglich ist. Die Adaptation bestehender Modelle, beispielsweise der motivierenden Gesprächsführung und deeskalierender Interaktionstechniken, konnte den bisher konzipierten, klassischen Shared-Decision-Making(SDM)-Ansatz erweitern und die Anpassung auf die Bedürfnisse dieser Patientengruppe weiter optimieren helfen. Gepaart mit fortlaufenden unterstützenden Schulungen der Patienten in der aktiven Teilnahme an Entscheidungsprozessen entsteht erstmals eine mehrdimensionale Empowerment-Strategie, die tatsächlich das Ausmaß der empfundenen Einbeziehung in Entscheidungsprozesse, die Zufriedenheit mit der Behandlung, das therapeutische Verhältnis und die Adhärenz gegenüber der vereinbarten medikamentösen Behandlungsstrategie verbessern kann. Diese positiven Effekte scheinen jedoch nach Verlassen der Klinik nicht weiter messbar fortzubestehen. Daher wäre eine behandlungssektorübergreifende Strategie in künftigen Studien hinsichtlich einer nachhaltigeren Verbesserung empfehlenswert. 
Interessenkonflikt

\section{Erklärung zu finanziellen Interessen}

Forschungsförderung erhalten: ja; Honorar/geldwerten Vorteil für Referententätigkeit erhalten: ja; Bezahlter Berater/interner Schulungsreferent/Gehaltsempfänger: ja; Patent/Geschäftsanteile/Aktien (Autor/Partner, Ehepartner, Kinder) an Firma (Nicht-Sponsor der Veranstaltung): nein; Patent/Geschäftsanteile/Aktien (Autor/Partner, Ehepartner, Kinder) an Firma (Sponsor der Veranstaltung): nein.

Erklärung zu nicht finanziellen Interessen

Die Autoren geben an, dass kein Interessenkonflikt besteht.

\section{Beratende Ethikkommission und} Studienregistrierung

Die Studie wurde von der Ethikkommission der Medizinischen Fakultät der Technischen Universität München beurteilt, alle Patienten gaben ihr schriftliches Einverständnis zur Studienteilnahme. Die Studie ist im Deutschen Register Klinischer Studien aufgeführt (DRKS00010880).

\section{Korrespondenzadresse}

\section{Prof. Dr. med. Stephan Heres}

kbo-Klinik für Psychiatrie und Psychotherapie Nord Schwabing der kbo-Tagesklinik und Institutsambulanz Nord Schwabing, Kölner Platz 1, Haus 7 80804 München, Deutschland Tel. 089/412006158 stephan.heres@kbo.de

\section{Literatur}

[1] Beitinger R, Kissling W, Hamann J. Trends and perspectives of shared decision-making in schizophrenia and related disorders. Current opinion in psychiatry 2014; 27(3): 222-9

[2] Drake RE, Deegan PE. Shared decision making is an ethical imperative. Psychiatric services 2009; 60(8): 1007

[3] Hamann J, Mendel RT, Fink B, et al. Patients' and psychiatrists' perceptions of clinical decisions during schizophrenia treatment. J Nerv Ment Dis 2008; 196(4): 329-32

[4] McCabe R, Khanom H, Bailey P, et al. Shared decision-making in ongoing outpatient psychiatric treatment. Patient education and counseling 2013; 91(3): 326-8

[5] Giacco D, Mavromara L, Gamblen J, et al. Shared decision-making with involuntary hospital patients: a qualitative study of barriers and facilitators. BJPsych Open 2018; 4(3): 113-8

[6] Hamann J, Kohl S, McCabe R, et al. What can patients do to facilitate shared decision making? A qualitative study of patients with depression or schizophrenia and psychiatrists. Soc Psychiatry Psychiatr Epidemiol 2016; 51(4): 617-25

[7] Legare F, Ratte S, Gravel K, et al. Barriers and facilitators to implementing shared decision-making in clinical practice: update of a systematic review of health professionals' perceptions. Patient education and counseling 2008; 73(3): 526-35

[8] Hamann J, Maris N, losifidou P, et al. Effects of a question prompt sheet on active patient behaviour: a randomized controlled trial with depressed outpatients. The International journal of social psychiatry 2014; 60(3): 227-35

[9] Hamann J, Langer B, Winkler V, et al. Shared decision making for in-patients with schizophrenia. Acta psychiatrica Scandinavica 2006; 114(4): 265-73

[10] Hamann J, Kohl S, McCabe R, et al. What can patients do to facilitate shared decision making? A qualitative study of patients with depression or schizophrenia and psychiatrists. Soc Psychiatry Psychiatr Epidemiol 2016; 51(4): 617-25

[11] Hamann J, Mendel R, Cohen R, et al. Psychiatrists' use of shared decision making in the treatment of schizophrenia: patient characteristics and decision topics. Psychiatric services 2009; 60(8): 1107-12

[12] Seale C, Chaplin R, Lelliott P, et al. Sharing decisions in consultations involving anti-psychotic medication: a qualitative study of psychiatrists' experiences. Social science \& medicine 2006; 62(11): 2861-73

[13] Hamann J, Heres S. Adapting Shared Decision Making for Individuals With Severe Mental Illness. Psychiatric services 2014; 65(12): 1483-6

[14] Priebe S, Richardson M, Cooney M, et al. Does the therapeutic relationship predict outcomes of psychiatric treatment in patients with psychosis? A systematic review. Psychotherapy and psychosomatics 2011; 80(2): 70-7

[15] Zolnierek KB, Dimatteo MR. Physician communication and patient adherence to treatment: a meta-analysis. Medical care 2009 ; 47(8): 826-34

[16] Hamann J, Holzhuter F, Stecher L, et al. Shared decision making PLUS - a cluster-randomized trial with inpatients suffering from schizophrenia (SDM-PLUS). BMC Psychiatry 2017; 17(1): 78

[17] Craig P, Dieppe P, Macintyre S, et al. Developing and evaluating complex interventions: the new Medical Research Council guidance. BMJ 2008; 337: a1655

[18] Hamann J, Parchmann A, Sassenberg N, et al. Training patients with schizophrenia to share decisions with their psychiatrists: a randomized-controlled trial. Soc Psychiatry Psychiatr Epidemiol 2017; 52(2): 175-82

[19] Elwyn G, Durand MA, Song J, et al. A three-talk model for shared decision making: multistage consultation process. BMJ2017; 359: j4891

[20] Hamann J, Mendel R, Meier A, et al. "How to speak to your psychiatrist": shared decision-making training for inpatients with schizophrenia. Psychiatric services 2011; 62(10): 1218-21

[21] Birchwood M, Smith ], Drury V, et al. A self-report Insight Scale for psychosis: reliability, validity and sensitivity to change. Acta psychiatrica Scandinavica 1994; 89(1): 62-7

[22] O’Donoghue B, Roche E, Ranieri V, et al. Service users' perceptions about their hospital admission elicited by service user-researchers or by clinicians. Psychiatric services 2013; 64(5): 416-22

[23] Rodenburg-Vandenbussche S, Pieterse AH, Kroonenberg PM, et al. Dutch Translation and Psychometric Testing of the 9-Item Shared Decision Making Questionnaire (SDM-Q-9) and Shared Decision Making Questionnaire-Physician Version (SDM-Q-Doc) in Primary and Secondary Care. PloS one 2015; 10(7): e0132158 
[24] McCabe R, Bullenkamp J, Hansson L, et al. The therapeutic relationship and adherence to antipsychotic medication in schizophrenia. PloS one 2012; 7(4): e36080

[25] Thompson K, Kulkarni J, Sergejew AA. Reliability and validity of a new Medication Adherence Rating Scale (MARS) for the psychoses. Schizophrenia research 2000; 42(3): 241-7

[26] Brähler E, Mühlan $\mathrm{H}$, Albani C, et al. Teststatistische Prüfung und Normierung der deutschen Versionen des EUROHIS-QOL Lebensqualität-Index und des WHO-5 Wohlbefindens-Index. Diagnostica 2007; 53(2): 83-96

[27] Kay SR, Wolkenfeld F, Murrill LM. Profiles of aggression among psychiatric patients. II. Covariates and predictors. J Nerv Ment Dis 1988; 176(9): 547-57

[28] Hamann J, John M, Holzhuter F, et al. Shared decision making, aggression, and coercion in inpatients with schizophrenia. Eur Psychiatry 2020; 63(1): e90

[29] Hamann J, Holzhuter F, Blakaj S, et al. Implementing shared decision-making on acute psychiatric wards: a cluster-randomized trial with inpatients suffering from schizophrenia (SDM-PLUS). Epidemiol Psychiatr Sci 2020; 29: e137
[30] Bramesfeld A, Klippel U, Seidel G, et al. How do patients expect the mental health service system to act? Testing the WHO responsiveness concept for its appropriateness in mental health care. Social science \& medicine 2007; 65(5): 880-9

[31] Burn E, Conneely M, Leverton M, et al. Giving Patients Choices During Involuntary Admission: A New Intervention. Front Psychiatry 2019; 10: 433

[32] Giacco D, Conneely M, Masoud T, et al. Interventions for involuntary psychiatric inpatients: A systematic review. Eur Psychiatry 2018; 54: 41-50

Bibliografie

Nervenheilkunde 2021; 40: 436-444

DOI 10.1055/a-1401-3586

ISSN 0722-1541

(c) 2021. Thieme. All rights reserved.

Georg Thieme Verlag KG, Rüdigerstraße 14,

70469 Stuttgart, Germany 


\section{Punkte sammeln auf CM/F.thieme.de}

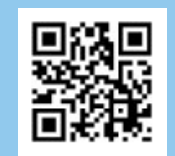

Diese Fortbildungseinheit ist bis zu 12 Monate online für die Teilnahme verfügbar.

Den genauen Einsendeschluss finden Sie beim Modul auf https://cme.thieme.de/CXGRKIE.

Sollten Sie Fragen zur Online-Teilnahme haben, finden Sie unter https://cme.thieme.de/hilfe

eine ausführliche Anleitung. Wir wünschen viel Erfolg beim Beantworten

der Fragen!

Unter https://eref.thieme.de/CXGRKIE oder über den QR-Code kommen Sie

direkt zum Artikel.

VNR 2760512021160211169

\section{Frage 1}

Mit dem Begriff „shared decision making“ (SDM) beschreibt man ...

A die gemeinsame Entscheidung von Patient und Angehörigen im Rahmen eines professionell angeleiteten Gespräches.

B die gemeinsame Entscheidung mehrerer medizinischer Fachdisziplinen in Form eines multidisziplinären Boards.

C die gemeinsame Entscheidung von gesetzlichem Betreuer und Vertretern des Behandlungsteam nach festgelegten Checklisten.

D die gemeinsame Entscheidung von Patient und Behandler nach beiderseitigem Austausch von Informationen zum Entscheidungsgegenstand.

E die Entscheidung des Patienten nach Durchsicht von virtuell aufbereiteten evidenzbasierten Informationsmaterialien.

\section{Frage 2}

Das Konzept des SDM ...

A wird von Patienten weitestgehend abgelehnt.

B wird von Psychiatern zu nahezu 95\% in allen Entscheidungssituationen angewandt.

$C$ ist in vielen Bereichen der Psychiatrie zwar allseits gewünscht, wird aber wenig angewandt.

D kann im psychiatrischen Setting aufgrund der eingeschränkten Krankheitseinsicht der Patienten, die an Schizophrenie leiden, kategorisch nicht angewandt werden.

E kann nur in Notfallsituationen angewandt werden.

\section{Frage 3}

SDMPLUS wiederum ...

A führt zu besseren Ergebnissen als klassisches SDM in allen klinischen Settings.

B ersetzt das klassische SDM komplett und sollte ausschließlich Anwendung finden.

C verwendet z. B. Techniken der motivationalen Gesprächsführung (motivational interviewing).

D ist bisher nur theoretisch verfügbar, aber wurde noch nie in einer Studie untersucht.

E existiert nur für andere Fachbereiche, aber nicht in der Psychiatrie.

\section{Frage 4}

SDMPLUS-Training umfasst ...

A Workshops für Angehörige von psychisch Erkrankten.

$B$ virtuelle Informationsangebote für Angehörige von psychisch Erkrankten.

C eModule mit Entscheidungsalgorithmen in der Antipsychotikatherapie.

D einen circa einjährigen Fachkurs.

E Patiententraining in Kommunikationstechniken sowie parallele Workshops für Behandlungsteams in den SDMPLUS-Techniken (double empowerment strategy).

\section{Frage 5}

In der beschriebenen SDMPLUS-Studie wurden Patienten ...

A im ambulanten Behandlungssetting untersucht.

B mit einer schweren Depression eingeschlossen.

C im tagklinischen Behandlungssetting einbezogen.

D im Rahmen eines akutpsychiatrischen vollstationären Aufenthaltes in einem geschützten Setting um ihre Teilnahme gebeten.

E mit der Grunderkrankung einer Schizophrenie ohne Einwilligung aufgrund der nicht gegebenen Einwilligungsfähigkeit in der Akutphase der Erkrankung mit Zustimmung der Ethikkommission erstmals rekrutiert.

\section{Frage 6}

An der Studie nahmen ...

A Patienten aus Kliniken in insgesamt 7 europäischen Ländern teil.

B Angehörige aus dem Landkreis München teil.

C Patienten und deren Angehörige aus den deutschsprachigen Ländern der EU teil.

D Patienten und Behandlungsteams von 12 Akutstationen aus 5 Kliniken teil.

E ausschließlich Trialoggruppen aus Angehörigen, Patienten und einem Arzt teil. 


\section{Punkte sammeln auf CME.thieme.de}

Fortsetzung ...

\section{Frage 7}

Die Randomisierung der Studie erfolgte ...

A doppelblind und placebokontrolliert.

B im Matching-Verfahren.

C durch ein Mirror-Image-Verfahren.

$\mathrm{D}$ in Form einer Einzelrandomisierung jedes teilnehmenden Patienten.

E als Cluster-Randomisierung, d. h. die Stationen wurden einmalig randomisiert, nicht aber die Teilnehmer selbst.

\section{Frage 8}

Die Ergebnisse der SDMPLUS-Studie zeigten zugunsten der Intervention ...

A eine statistisch signifikant erhöhte subjektiv erlebte Einbeziehung der Teilnehmer in Entscheidungsprozesse.

B deutlich niedrigere Rehospitalisierungsraten in der Postinterventionsphase.

C eine Verdoppelung der Compliance mit der verordneten Medikationseinnahme nach Entlassung aus der stationären Behandlung.

D eine messbare Zunahme der therapeutischen Allianz bis zum Endpunkt der Nachbeobachtung nach 12 Monaten.

E eine Zunahme der Diskussionsdauer der Entscheidungsfindungsprozesse während des stationären Aufenthaltes um circa $30 \%$.

\section{Frage 9}

Das Messinstrument für den primären Endpunkt der Studie war die ...
A PANSS-Skala.
B CGI-S-Skala.
C MARS-Skala.
D PSP-Skala.
E SDM-Q-9-Skala.

\section{Frage 10}

Die Intervention wirkte sich ...

A auf Patienten, die entgegen ihrem Willen in die stationäre Behandlung aufgenommen worden waren, hinsichtlich der erlebten Einbeziehung in Entscheidungsprozesse in etwa vergleichbar aus wie auf die freiwillig stationär aufgenommenen Teilnehmer.

B auf Patienten, die dokumentierte Aggressionshandlungen aufwiesen, besonders effektiv aus.

C auf Patienten, die Zwangsbehandlungen in der Präinterventionsphase erlebt hatten, in besonders hohem Maße aus.

D bei allen Patienten mit einer Verkürzung der Dauer bis zum ersten Alleinausgang von der geschützten Station aus.

E vor allem in der Postinterventionsphase bei Patienten mit dokumentierten freiheitsentziehenden Maßnahmen in Form einer signifikant erhöhten therapeutischen Allianz aus. 